\title{
Limits on the Extent of the Solsticial Hadley Cell: The Role of Planetary Rotation
}

\author{
MARTIN S. SINGH \\ School of Earth, Atmosphere and Environment, and Centre of Excellence for Climate Extremes, \\ Monash University, Clayton, Victoria, Australia
}

(Manuscript received 23 November 2018, in final form 17 April 2019)

\begin{abstract}
The role of planetary rotation in limiting the extent of the cross-equatorial solsticial Hadley cell (SHC) is investigated using idealized simulations with an aquaplanet general circulation model run under perpetualsolstice conditions. Consistent with previous studies that include a seasonal cycle, the SHC extent increases with decreasing rotation rate, and it occupies the entire globe for sufficiently low planetary rotation rates. A simple theory for the summer-hemisphere extent of the SHC is constructed in which it is assumed that the SHC occupies regions for which a hypothetical radiative-convective equilibrium state is physically unattainable. The theory predicts that the SHC extends farther into the summer hemisphere as the rotation rate is decreased, qualitatively reproducing the behavior of the simulations, but it generally underestimates the simulated SHC extent. A diagnostic theory for the summer-hemisphere SHC extent is then developed based on the assumptions of slantwise convective neutrality and conservation of angular momentum within the Hadley cell. The theory relates the structure of the SHC in the summer hemisphere to the distribution of boundary layer entropy in the dynamically equilibrated simulations. The resultant diagnostic for the SHC extent generalizes the convective quasi-equilibrium-based constraint of Privé and Plumb, in which the position of rain belts is related to maxima in the low-level entropy distribution.
\end{abstract}

\section{Introduction}

The seasonal cycle of Earth's zonal-mean tropical circulation is characterized by a transition from an equinoctial regime, comprising a pair of Hadley cells of roughly equal strength, to a solsticial regime dominated by a single cross-equatorial cell with a rising branch in the summer hemisphere (Dima and Wallace 2003). This transition is associated with a poleward shift of the intertropical convergence zone (ITCZ) and the onset of monsoons over tropical continents (Bordoni and Schneider 2008). The latitudinal extent of the cross-equatorial solsticial Hadley cell (SHC) is therefore a key determinant of the distribution of precipitation in many tropical and subtropical regions.

The ultimate driver of the seasonal rearrangement of the tropical circulation is the variation in solar insolation associated with Earth's orbit around the sun. But while the solsticial peak in daily mean top-of-atmosphere solar insolation occurs at the summer pole, the rising branch of Earth's Hadley cell remains within the tropics and subtropics throughout the year. One obvious reason that

Corresponding author: Martin S. Singh, martin.singh@monash.edu the Hadley cell's rising branch does not simply "follow the sun" is that the atmosphere and surface have nonnegligible thermal inertia. Indeed, observations and general circulation model (GCM) simulations indicate that tropical rain belts shift farther into the summer hemisphere over surfaces with lower thermal heat capacity (e.g., Wang and Ding 2008; Bordoni and Schneider 2008; Donohoe et al. 2014). However, Faulk et al. (2017) has recently shown that, in idealized simulations with a moist GCM, the rising branch of the SHC remains at subtropical latitudes even when allowed to equilibrate under perpetual-solstice forcing. Under such forcing, the effects of thermal inertia on the mean circulation are absent, highlighting the influence of other factors, such as the planetary rotation rate, on the position and extent of the SHC.

Previous studies applying perpetual-solstice forcing within a dry framework have shown that the SHC widens as the planetary rotation rate is decreased (Caballero 2008; Hill et al. 2019), but a quantitative theory for its extent remains elusive. In this work, we focus on this perpetual-solstice case within a moist framework in order to isolate the role played by planetary rotation in determining the SHC extent and the resultant distribution 
of precipitation. Understanding this limiting case is a prerequisite for developing a theory for the full seasonal cycle of the tropical circulation.

A useful starting point for theoretical discussions of the Hadley cell is the axisymmetric nearly inviscid models pioneered by Schneider (1977) and Held and Hou (1980). While such models neglect the effect of eddy momentum fluxes on the mean circulation (Walker and Schneider 2006; Caballero 2007, 2008; Singh and Kuang 2016; Singh et al. 2017), these effects are less important for the SHC than its equinoctial counterpart (Bordoni and Schneider 2008). In the nearly inviscid limit, the combination of angular momentum conservation and thermal wind balance within the free troposphere places a strong constraint on the thermodynamic structure of the atmosphere. If it is further assumed that the Hadley cells are energetically closed, a prediction for the width of the cells and, in the case of an off-equatorial forcing maximum, the position of the rising branch may be derived (Lindzen and Hou 1988). However, Caballero et al. (2008) found that, for the case in which the thermal forcing maximizes at the pole, nearly inviscid theory substantially overestimates the width of the SHC compared to axisymmetric simulations with a GCM. The authors instead derived a semiempirical scaling for the latitudinal extent of the SHC's descending branch, but no theoretical constraint on the position of the rising branch was obtained.

Axisymmetric theory may also be used to investigate the onset conditions for large-scale thermally direct circulations. For example, Plumb and Hou (1992) derived a critical threshold for the strength of an isolated, offequatorial thermal forcing maximum beyond which a hypothetical radiative-convective equilibrium (RCE) state becomes unattainable. Under the approximation of convective quasi equilibrium (Emanuel et al. 1994), this critical threshold may be expressed in terms of the boundary layer entropy distribution of the RCE state (Emanuel 1995). In principle, such a criticality condition could provide a constraint on the extent of the SHC, since an overturning circulation must extend at least over the region for which the RCE state is unattainable. One of the aims of this work is to test the applicability of this criticality condition to the solsticial circulation (see also Faulk et al. 2017; Hill et al. 2019).

A number of authors have also used diagnostic approaches in order to relate the position of the Hadley cell's rising branch to atmospheric energy transport characteristics (e.g., Kang et al. 2008, 2009; Donohoe et al. 2013; Bischoff and Schneider 2014; Wei and Bordoni 2018) or local thermodynamic properties of the atmosphere (e.g., Lindzen and Nigam 1987; Neelin and Held 1987; Back and Bretherton 2009a,b; Nie et al. 2010).
For instance, Privé and Plumb (2007a,b) found that the dividing streamline between summer and winter Hadley circulations was roughly collocated with the maximum in low-level moist static energy in simulations of an idealized monsoon circulation, with the maximum in convergence occurring somewhat equatorward of this location. More generally, the convective quasi-equilibrium view of the tropical circulation (Emanuel et al. 1994) argues that tropical precipitation belts should lie close to local maxima of boundary layer moist static energy or the related quantity of moist entropy (Neelin and Held 1987; Nie et al. 2010). But as pointed out by Faulk et al. (2017), the maximum in boundary layer entropy becomes increasingly separated from the ITCZ and the Hadley cell edge as these quantities move poleward. Indeed, the authors find that, in perpetual-solstice simulations with Earthlike parameters, the maximum in boundary layer entropy occurs at the pole, but the rising branch of the SHC remains at subtropical latitudes.

A limitation of the convective quasi-equilibrium viewpoint is that, under conditions of strong vertical wind shear, it predicts a state of moist symmetric instability, in which potential energy may be released by the motion of saturated parcels along slantwise paths oriented along angular momentum surfaces (Emanuel 1983a,b). Such slantwise convection has been recognized as being important for the structure of both tropical (Emanuel 1986) and extratropical (Emanuel 1988) cyclones, but its importance in determining the character of large-scale overturning circulations is largely unknown.

Here, we build on the study of Faulk et al. (2017), and we seek to understand the factors limiting the extent of the SHC under conditions where the thermal maximum is located at the summer pole. We conduct simulations with an idealized moist GCM forced by perpetualsolstice conditions over a range of planetary rotation rates. The simulated SHC extent decreases with increasing rotation rate, despite the fact that the highest boundary layer entropy values remain at the summer pole. These results are interpreted by constructing a predictive theory for the summer-hemisphere SHC extent based on the criticality constraint of Emanuel (1995) and a diagnostic theory based on slantwise convective neutrality within the Hadley cell. The diagnostic theory relates the summer-hemisphere SHC edge latitude to the boundary layer entropy distribution, generalizing previous constraints on tropical precipitation based on convective quasi equilibrium.

We first present the model configuration (section 2) and the basic characteristics of the simulated SHC (section 3). We then describe the predictive (section 4) and diagnostic (section 5) theories of the summer-hemisphere 
SHC extent and compare them to the idealized simulations. Finally, we present a summary and discussion (section 6).

\section{Simulation design}

We conduct simulations under perpetual-solstice conditions using an idealized aquaplanet GCM similar to that of Frierson et al. (2006, 2007). The model is based on the Geophysical Fluid Dynamics Laboratory Flexible Modeling System, and it includes a two-stream semigray radiation scheme and a representation of moisture with a single vapor-liquid phase transition. Additionally, the model employs the simplified quasiequilibrium convection scheme described in Frierson (2007), a saturation adjustment scheme to prevent gridscale supersaturation, and a $k$-profile boundary layer parameterization similar to that of Troen and Mahrt (1986). The surface is assumed to be a slab ocean with a fixed depth of $2 \mathrm{~m}$, and surface fluxes are computed based on bulk aerodynamic formulas, with transfer coefficients calculated based on Monin-Obukhov similarity theory.

Details of our model configuration follow those of O'Gorman and Schneider (2008) except that 1) the model is forced using a solar insolation profile characterized by diurnally averaged conditions at the Northern Hemisphere summer solstice and 2) we do not allow the longwave optical depth to depend on latitude. In particular, the top-of-atmosphere solar insolation $S_{\mathrm{TOA}}$ is given as a function of latitude $\phi$ by (Hartmann 1994, p. 30),

$$
S_{\mathrm{TOA}}=\frac{S_{0}}{\pi}\left(h_{0} \sin \phi \sin \delta+\cos \phi \sin h_{0}\right)
$$

where the declination angle $\delta$ is equal to Earth's axial tilt of $23.4^{\circ}$, we set $S_{0}=1367 \mathrm{~W} \mathrm{~m}^{-2}$, and $h_{0}$ is defined by

$$
\cos h_{0}=\left\{\begin{aligned}
1, & \phi & \leq \delta-\frac{\pi}{2}, \\
-\tan \phi \tan \delta, & \delta-\frac{\pi}{2} & <\phi<\frac{\pi}{2}-\delta, \\
-1, & \phi & \geq \frac{\pi}{2}-\delta .
\end{aligned}\right.
$$

This imposed insolation profile is shown in Fig. 1; note that the insolation is zero poleward of the Antarctic circle at $66.6^{\circ} \mathrm{S}$. Atmospheric absorption of solar radiation is treated as in O'Gorman and Schneider (2008) such that the atmosphere absorbs roughly $20 \%$ of the incoming solar radiation, and the surface albedo is set

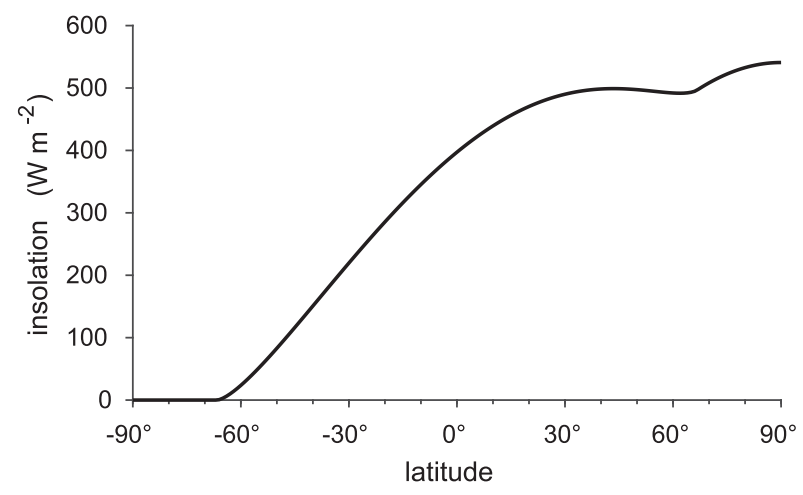

FIG. 1. Top-of-atmosphere solsticial insolation profile defined by (1) used to force the GCM simulations.

to 0.38 , with all reflected radiation emitted directly to space.

The longwave optical depth $\tau$ is specified as a function of the model's vertical sigma coordinate so that

$$
\tau=\tau_{s}\left[f \sigma+(1-f) \sigma^{4}\right] .
$$

Here, $\sigma=p / p_{s}$ is the pressure $p$ normalized by the surface pressure $p_{s}$, and, following O'Gorman and Schneider (2008), we set $f=0.2$. The optical depth at the surface $\tau_{s}$ determines the overall strength of the greenhouse effect, and we set it to $\tau_{s}=3.5$ to obtain surface temperatures roughly similar to present-day Earth. (The mean summer-hemisphere surface temperature varies between 289 and $307 \mathrm{~K}$ across the simulations.) In the perpetualequinox simulations of O'Gorman and Schneider (2008), the longwave optical depth $\tau_{s}$ was prescribed to vary in latitude, with a maximum at the equator to mimic the increased greenhouse effect associated with high water vapor concentrations at low latitudes. In our solsticial simulations, the water vapor concentration generally peaks near the summer pole, and a equatorial maximum in $\tau_{s}$ is inappropriate. For simplicity, we instead follow Faulk et al. (2017) and set $\tau_{s}$ to a constant.

We conduct a series of 11 perpetual-solstice simulations in which the planetary rotation rate is varied from $\Omega_{e} / 8$ to $8 \Omega_{e}$, where $\Omega_{e}=7.29 \times 10^{-5} \mathrm{~s}^{-1}$ is Earth's rotation rate. The simulations are run at T42 spectral resolution with 30 unevenly spaced sigma levels in the vertical for 6 years $(1$ year $=360$ days $)$ from an isothermal initial state; the final 2 years of the simulations are used to construct time-averaged statistics.

\section{Simulated precipitation and circulation}

Figure 2 shows snapshots of near-surface temperature, column water vapor, and precipitation from simulations 

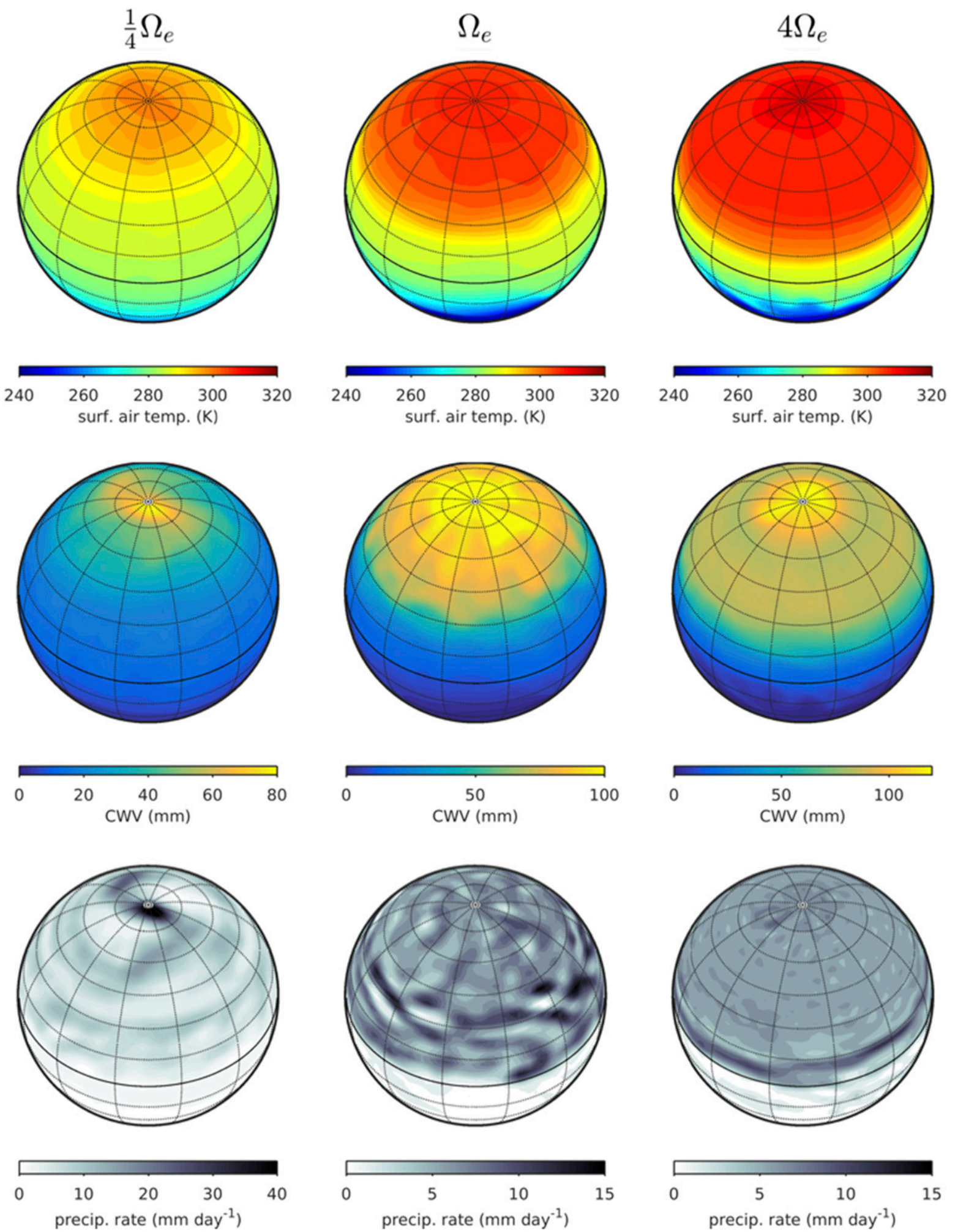

FIG. 2. Snapshots of (top) temperature at the lowest model level, (middle) column water vapor, and (bottom) precipitation rate for simulations with rotation rates $\Omega$ equal to (left) $\Omega_{e} / 4$, (center) $\Omega_{e}$, and (right) $4 \Omega_{e}$. 

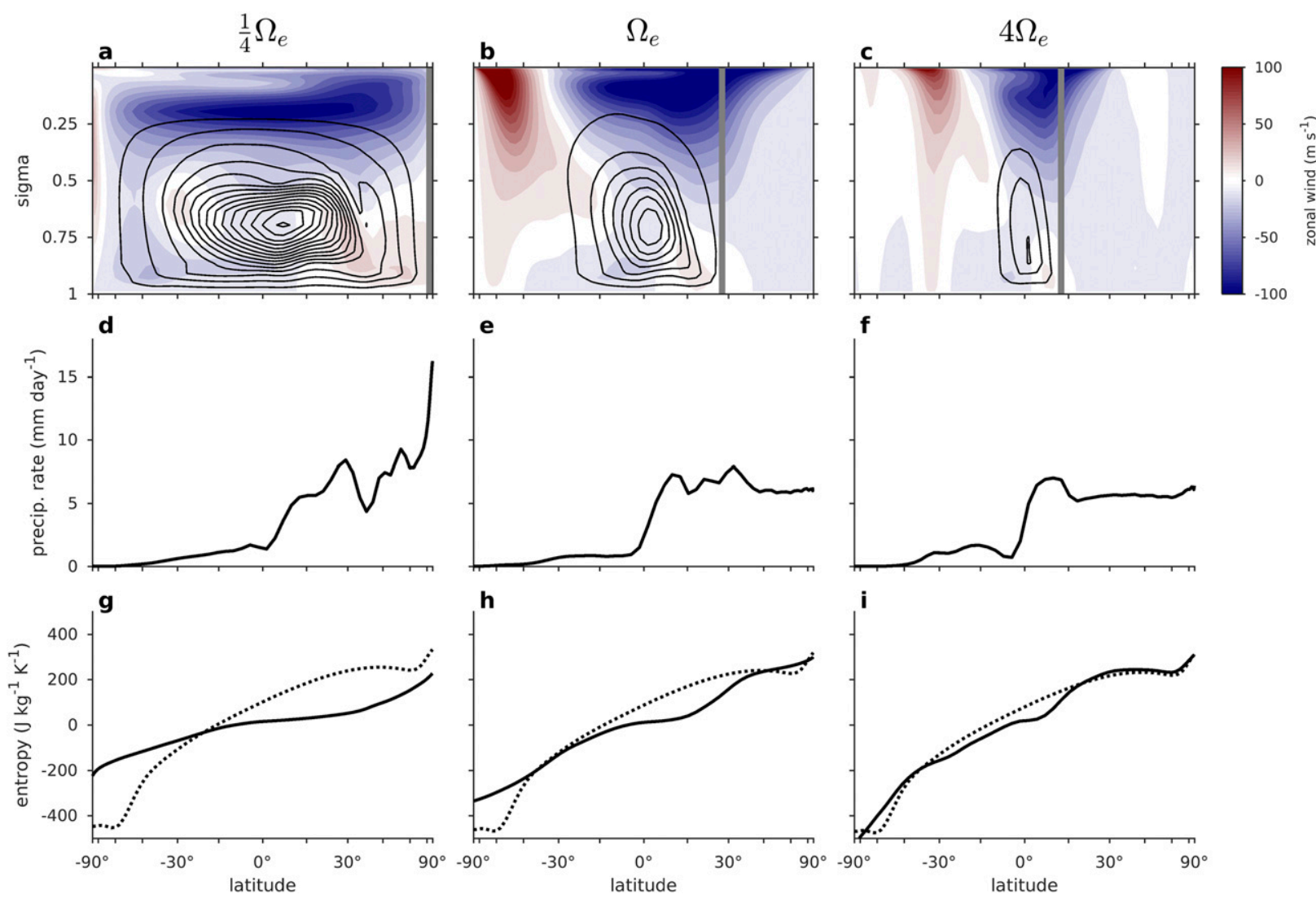

FIG. 3. (a)-(c) Streamfunction [contours; contour interval (CI) $=10^{11} \mathrm{~kg} \mathrm{~s}^{-1}$ ] and zonal- and time-mean zonal wind (colors), (d)-(f) zonal- and time-mean precipitation rate, and (g)-(i) zonal- and time-mean boundary layer entropy $s_{b}$ (adjusted to have zero global mean) as a function of sine latitude for simulations with planetary rotation rates equal to (left) $\Omega_{e} / 4$ (center) $\Omega_{e}$ and (right) $4 \Omega_{e}$. Gray vertical lines in (a)-(c) show latitude of the summer-hemisphere SHC edge $\phi_{h}$, and dotted lines in (g)-(i) show $s_{\mathrm{RCE}}$, the boundary layer entropy distribution in the RCE simulation.

under three different rotation rates. As expected from the imposed insolation profile, near-surface temperatures are generally highest at the north (summer) pole in all simulations, with relatively weak gradients in the Northern Hemisphere. As a result of the strong relationship between temperature and saturation vapor pressure, column water vapor values also peak at the North Pole and decrease toward the south. The latitude of the highest precipitation rates, however, decreases with increasing rotation rate from the pole in the $\Omega=\Omega_{e} / 4$ case to only a few degrees north of the equator in the $\Omega=4 \Omega_{e}$ simulation.

The picture above is confirmed in the time and zonal mean; the latitude of the maximum in the zonal- and time-mean precipitation, which we define as $\phi_{P}$, shifts from the North Pole in the $\Omega=\Omega_{e} / 4$ simulation to roughly $10^{\circ} \mathrm{N}$ as the rotation rate is increased to $4 \Omega_{e}$ (Figs. 3d-f). This shift is accompanied by a contraction of the cross-equatorial SHC from a global pole-to-pole circulation in the $\Omega=\Omega_{e} / 4$ case to a weaker circulation occupying only a few degrees of latitude for $\Omega=4 \Omega_{e}$ (Figs. 3a-c). In all our simulations, the cross-equatorial SHC dominates the zonal-mean overturning; in cases where the SHC is not global, a weak eddy-driven Ferrel cell exists poleward of the SHC the winter hemisphere, but the summer Hadley cell and the Ferrel cell in the summer hemisphere are absent. Associated with the SHC, strong easterlies, in excess of $100 \mathrm{~m} \mathrm{~s}^{-1}$, exist in the tropical upper troposphere for all values of $\Omega$ simulated (Figs. 3a-c). For the slowly rotating case $\left(\Omega=\Omega_{e} / 4\right)$, these easterlies extend globally throughout the upper troposphere. For more rapidly rotating cases, uppertropospheric westerlies are present beyond the southern edge of the SHC.

As the rotation rate is increased from $\Omega_{e} / 8$ to $8 \Omega_{e}$, the precipitation distribution shifts equatorward, but the shift of $\phi_{P}$ itself is not monotonic (Fig. 4). This is because there are typically two peaks in the zonal- and time-mean precipitation, one that remains relatively close to the equator and one that is located close to the 


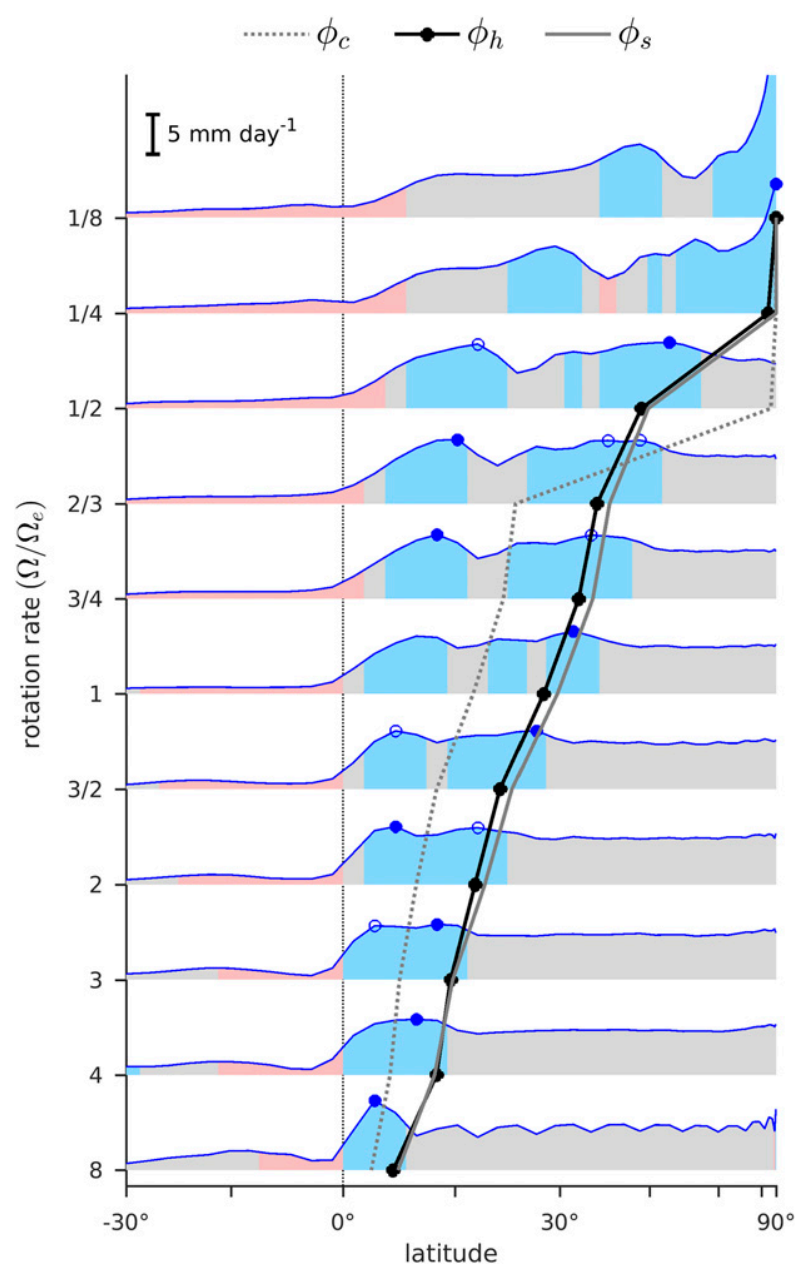

FIG. 4. Zonal- and time-mean precipitation rate as a function of sine latitude for simulations with different rotation rates as labeled (blue lines; each curve offset by $12 \mathrm{~mm}$ day $^{-1}$ ). Regions in which the ratio of precipitation to evaporation $P / E>1.2$ are shaded blue, regions in which $P / E<0.8$ are shaded pink, and all other regions are shaded gray. Blue symbols indicate local maxima in precipitation with magnitudes greater than $95 \%$ of the global maximum; the global maximum is shown by a filled symbol. Black and gray lines show latitude of the SHC edge in the summer hemisphere as simulated by the GCM $\phi_{h}$ (black line with symbols), as predicted based on supercriticality of the RCE state $\phi_{c}$ (gray dotted line), and as predicted by the slantwise neutral solution $\phi_{s}$ (gray solid line). Figure inspired by Shekhar and Boos (2016).

edge of the Hadley cell, and their relative magnitude varies as a function of the rotation rate. In contrast, the latitudinal extent of the SHC into the summer hemisphere does increase monotonically with rotation rate (black line with symbols in Fig. 4). Here, the latitude of the SHC edge in the Northern (summer) Hemisphere $\phi_{h}$ is defined based on the location at which the magnitude of the streamfunction $\Psi$ is reduced below a threshold. Specifically, we define $\phi_{h}$ as the first latitude north of the streamfunction maximum that satisfies

$$
\frac{\Psi\left(\phi_{h}, \sigma_{\max }\right)}{\cos \phi_{h}}=0.1 \frac{\Psi_{\max }}{\cos \phi_{\max }}
$$

where $\phi_{\max }$ and $\sigma_{\max }$ are, respectively, the latitude and sigma level at which the streamfunction magnitude reaches its maximum value $\Psi_{\max }$. The cosine factors are included in (2) to account for the reduction in $\Psi$ associated with the geometry of Earth; for rotation rates below $\Omega_{e} / 2$, the maximum in zonal- and time-mean precipitation occurs at the summer pole, but unless the cosine factors are included, the diagnosed Hadley cell extent is equatorward of the region of strongest ascent. For rotation rates greater than $\Omega_{e} / 2$, the cosine factors have a very small effect $\left(<2.5^{\circ}\right)$ on the diagnosed value of $\phi_{h}$.

Given the sensitivity of the position of the precipitation maximum to small changes in the precipitation distribution, we focus here on understanding the effect of planetary rotation on the latitude of the SHC edge $\phi_{h}$ rather than on the latitude of maximum precipitation $\phi_{P}$. In all our simulations, $\phi_{h}$ is within a few degrees of a local maximum in the zonal- and time-mean precipitation distribution corresponding to the rising branch of the Hadley cell, and $\phi_{h}$ roughly demarcates the boundary between the region of moisture convergence within the Hadley cell rising branch and the region in which the precipitation and evaporation are roughly in balance farther poleward (Fig. 4).

The contraction of the SHC and equatorward shift of the precipitation distribution with increasing rotation rate occur in our simulations despite the fact that the zonal- and time-mean boundary layer entropy, $s_{b}$, is maximized at the summer pole for all rotation rates (Figs. 3g-i). ${ }^{1}$ This is in contrast to many previous studies that have argued that tropical precipitation maxima should lie adjacent to maxima in boundary layer entropy, or the closely related quantity moist static energy (e.g., Neelin and Held 1987; Privé and Plumb 2007a,b; Nie et al. 2010). Such arguments generally rely on the assumption of convective quasi equilibrium and either the application of the weak temperature gradient (WTG) approximation (Sobel et al. 2001) to the region of strong convection in the ITCZ (Nie et al. 2010) or the assumption that the rising

\footnotetext{
${ }^{1}$ Here $s_{b}$ is calculated based on the time- and zonal-mean distributions of temperature, pressure, and mixing ratio at the lowest model level. Similar results are obtained if an average over levels below $\sigma=0.8$ is used instead.
} 
branch of the SHC is in a region of weak wind shear (Privé and Plumb 2007a). In our simulations, the rising branch of the SHC exists in a region of strong easterly shear and, by thermal wind balance, a region with substantial meridional gradients of temperature in the free troposphere, and thus the argument of Privé and Plumb (2007a) is inapplicable. Understanding the contraction of the SHC and the increasing separation of the SHC edge from the maximum in boundary layer entropy with increasing planetary rotation rate is the aim of the next two sections.

\section{Predictive estimate of the summer-hemisphere SHC extent}

To construct a theory for the latitude $\phi_{h}$, we first consider the behavior of the atmosphere in the absence of large-scale circulations. Under these conditions, each column of the atmosphere is separately in RCE, and the zonal flow may be assumed to be in gradient wind balance with the resultant temperature field. As pointed out by Hide (1969), such a state is unattainable if it produces a maximum in angular momentum above the surface, as such a maximum cannot be maintained against vertical momentum diffusion (see also Schneider 1977; Held and Hou 1980). A sufficient condition for the existence of an elevated angular momentum maximum is that the absolute vorticity in the RCE state change sign within a hemisphere (Plumb and Hou 1992). Under the assumption of convective quasi equilibrium, in which the boundary layer entropy is directly tied to the free-tropospheric saturation entropy, this condition may be written as (Emanuel 1995)

$$
-\frac{1}{\sin \phi} \frac{\partial}{\partial \phi}\left(\frac{\cos ^{3} \phi}{\sin \phi} \Delta T_{E} \frac{\partial s_{E}}{\partial \phi}\right)>4 \Omega^{2} R_{e}^{2} \cos ^{3} \phi
$$

where $s_{E}$ is the boundary layer entropy distribution and $\Delta T_{E}$ is the temperature difference between the surface and the tropopause, both measured in the RCE state, and $R_{e}$ is Earth's radius.

Equation (3) defines a criticality condition on the boundary layer entropy distribution; when $s_{E}$ is supercritical, (3) is satisfied and the RCE state is unattainable, implying the existence of a large-scale overturning circulation. In particular, (3) is satisfied if the gradient of $s_{E}$ is nonzero at the equator, a condition virtually guaranteed in our perpetual-solstice case. Farther poleward, however, the supercriticality of the RCE state depends on the curvature of $s_{E}$; indeed the satisfaction of (3) in the subtropics has been argued to be a relevant condition for the onset of monsoons (Plumb and Hou 1992).
To construct a simple predictive estimate for the summer-hemisphere extent of the SHC, we assume that, in the summer hemisphere, the Hadley cell extends only over latitudes for which $s_{E}$ is supercritical according to (3). This estimate is predictive because it depends only on the RCE state, which in principle may be calculated independently of the full dynamical equilibrium. In practice, the RCE state is taken as the zonal and time mean of a simulation conducted using the same model configuration as used for the full dynamical simulations, but with no meridional circulation or lateral transports allowed. ${ }^{2}$ The temperature distribution in this RCE simulation is therefore a result of the interaction of the radiation, convection, boundary layer, and surface-flux parameterizations within each column of the GCM, but it does not depend appreciably on the planetary rotation rate. ${ }^{3}$

The near-surface temperature of the RCE simulation mimics the top-of-atmosphere insolation profile, with strong gradients in the Southern Hemisphere and a weak maximum at the North Pole (Fig. 5a). As expected from the quasi-equilibrium convection scheme used in the simulations, the tropospheric lapse rate in the RCE simulation is close to moist adiabatic, and contours of saturation entropy are nearly vertical within the free troposphere (Fig. 5b). To evaluate the criticality criterion (3), we must estimate $\Delta T_{E}$, the depth of the troposphere in temperature coordinates. Here, we consider the upper boundary of the troposphere to be the highest level to which convection has a strong influence on the RCE temperature profile. This level is clearly demarcated in Fig. 5b by the location at which saturation entropy contours rapidly switch from being primarily vertically oriented to being primarily horizontally oriented. We find a good estimate of this level to be the height at which the lapse rate drops below half of its moist-adiabatic value, and this gives $\Delta T_{E} \approx 50 \mathrm{~K}$ throughout the Northern Hemisphere. ${ }^{4}$ Applying the approximation of constant $\Delta T_{E}$ to (3), we may write the criticality condition as (Emanuel 1995)

\footnotetext{
${ }^{2}$ To ensure an equilibrium is reached, the solsticial insolation profile is altered from that used in the full dynamical simulations such that the top-of-atmosphere insolation does not drop below $20 \mathrm{~W} \mathrm{~m}^{-2}$ in the RCE simulation. This alteration only affects the solution in the high southern latitudes.

${ }^{3}$ We follow O'Gorman and Schneider (2008) and use the zonaland time-mean wind field from the equilibrated simulation with $\Omega=\Omega_{e}$ as an input to the boundary layer and surface-flux schemes, but in all other respects, the RCE simulation does not depend on the planetary rotation rate.

${ }^{4}$ Using a threshold lapse rate of $2 \mathrm{~K} \mathrm{~km}^{-1}$ (as commonly used to define the tropopause) gives a slightly larger value for $\Delta T_{E}$, but this does not appreciably affect the predicted SHC extent $\phi_{c}$.
} 

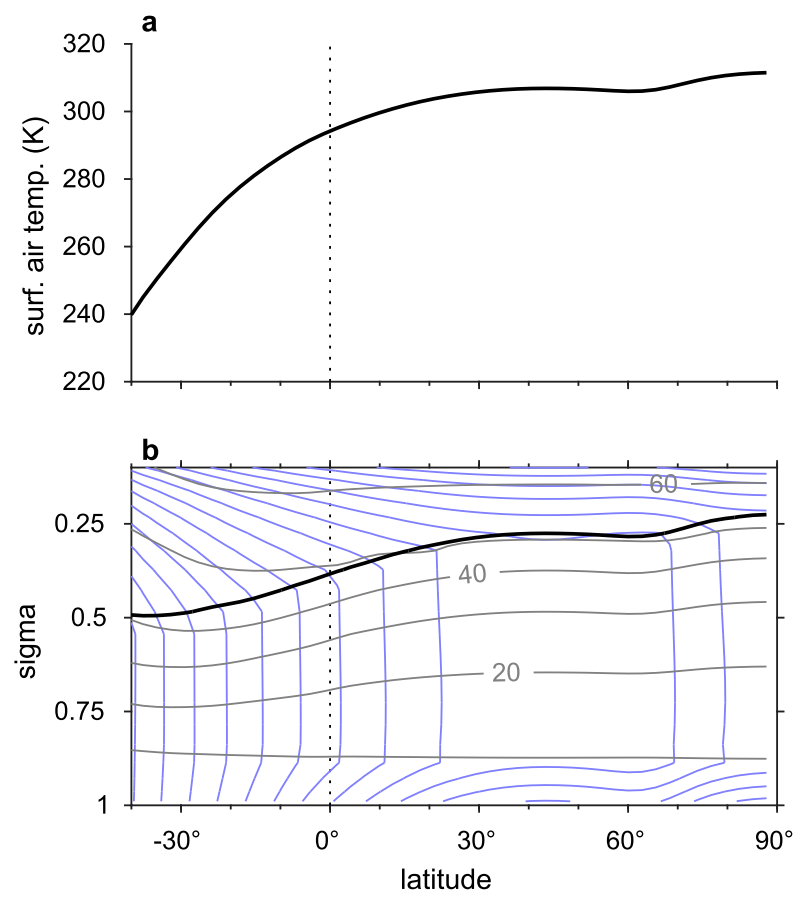

FIG. 5. RCE solution for solsticial insolation profile. (a) Temperature at the lowest model level. (b) Saturation entropy (blue; contour interval $50 \mathrm{~J} \mathrm{~kg}^{-1} \mathrm{~K}^{-1}$ ) and temperature anomaly relative to temperature at the lowest model level (gray). Black line in (b) shows the tropopause level, defined as the height at which the lapse rate decreases to half of its moist-adiabatic value.

$$
-\frac{\partial^{2} \hat{s}_{E}}{\partial y^{2}}>\frac{1}{y^{3}}\left(\frac{\Omega}{\Omega_{e}}\right)^{2},
$$

where $y=\sec ^{2} \phi$, and we have nondimensionalized the entropy so that $\hat{s}_{E}=\Delta T_{E} s_{E} / \Omega_{e}^{2} R_{e}^{2}$.

Figure 6 shows the two sides of (4) for three different rotation rates. Since the RCE entropy distribution is independent of rotation rate, the curvature $\partial^{2} s_{E} / \partial y^{2}$ is fixed, but the critical entropy curvature, given by the right-hand side of (4) increases quadratically with the planetary rotation rate. Thus, while (4) is satisfied at the equator for all rotation rates, the latitude at which the RCE solution becomes subcritical increases with decreasing rotation rate. For the most slowly rotating cases (e.g., $\Omega=\Omega_{e} / 2$ ), $s_{E}$ becomes supercritical in two separate regions, one near the equator and one at polar latitudes. Here, we assume that the Hadley cell extends to the poleward most region of supercriticality based on our expectation that the Hadley cell should become global in the limit $\Omega \rightarrow 0$.

The dotted gray line in Fig. 4 shows the supercriticalitybased prediction of the summer-hemisphere SHC edge, which we denote $\phi_{c}$, for different rotation rates. As the rotation rate is increased, $\phi_{c}$ decreases, consistent with the behavior of the SHC extent in the simulations

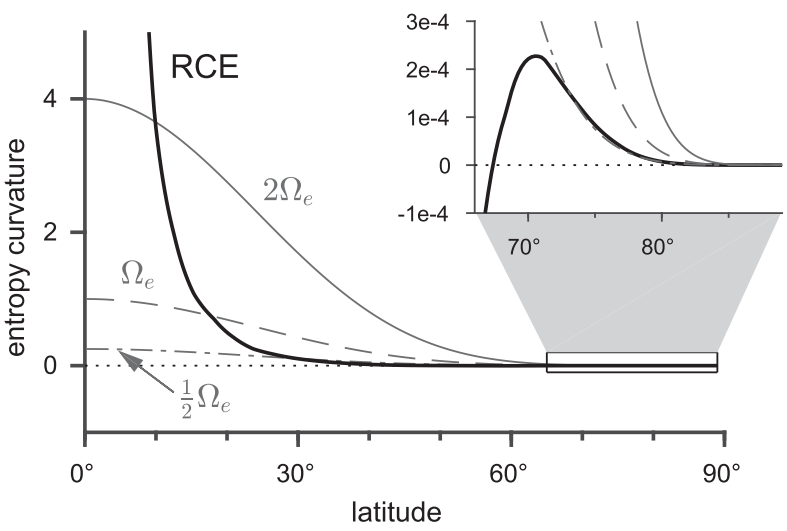

FIG. 6. Supercriticality of the RCE distribution of boundary layer entropy $s_{E}$. Black line shows the entropy curvature [left-hand side of (4)] and gray lines show the critical value of this curvature [righthand side of (4)] for various values of the planetary rotation rate.

(see also Fig. 10). However, at most rotation rates, $\phi_{c}$ tends to underestimate the summer-hemisphere SHC extent. Thus, while the predictive estimate of the SHC extent $\phi_{c}$ provides a qualitative explanation for the changes in the Hadley cell extent with increasing rotation rate, it does not provide a quantitatively accurate theory.

A potential reason that $\phi_{c}$ tends to underestimate the summer-hemisphere SHC extent is that the criticality condition (3) only demarcates regions in which a largescale circulation is required in order to satisfy Hide's constraint; it does not preclude the Hadley cell from extending beyond the region of supercriticality. We may therefore more sensibly view this criticality-based estimate as a lower bound on the latitude $\phi_{h}$ rather than a precise prediction; indeed, $\phi_{c} \lesssim \phi_{h}$ in all but one of the simulations. ${ }^{5}$ These results are consistent with those of Faulk et al. (2017), who found that a similar supercriticality condition underestimated the latitude of the solsticial ITCZ position in idealized aquaplanet GCM simulations. Further discussion of the limitations of estimates of Hadley cell extent based on violation of Hide's constraint in the RCE state may also be found in Hill et al. (2019).

\section{Diagnostic estimate of summer-hemisphere SHC extent}

To obtain a more accurate estimate of the SHC extent $\phi_{h}$, we consider how the circulation itself influences the

\footnotetext{
${ }^{5}$ The exception is the case $\Omega=\Omega_{e} / 2$, which represents a transition point between cases where the RCE state has only one region of supercriticality in the tropics and cases where there is a second region of supercriticality in subpolar latitudes (Fig. 6).
} 

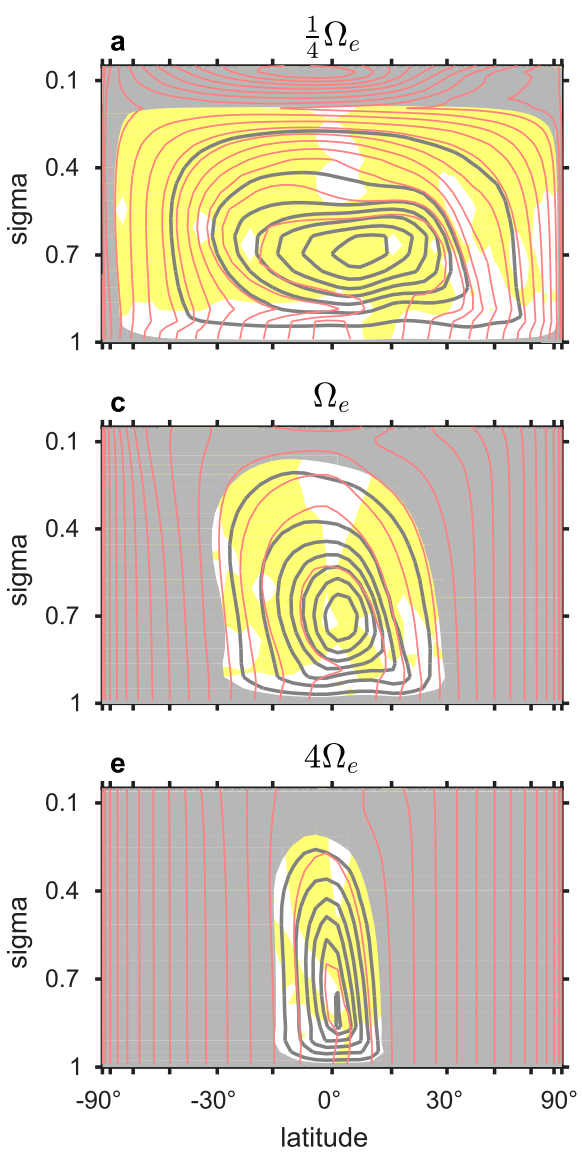
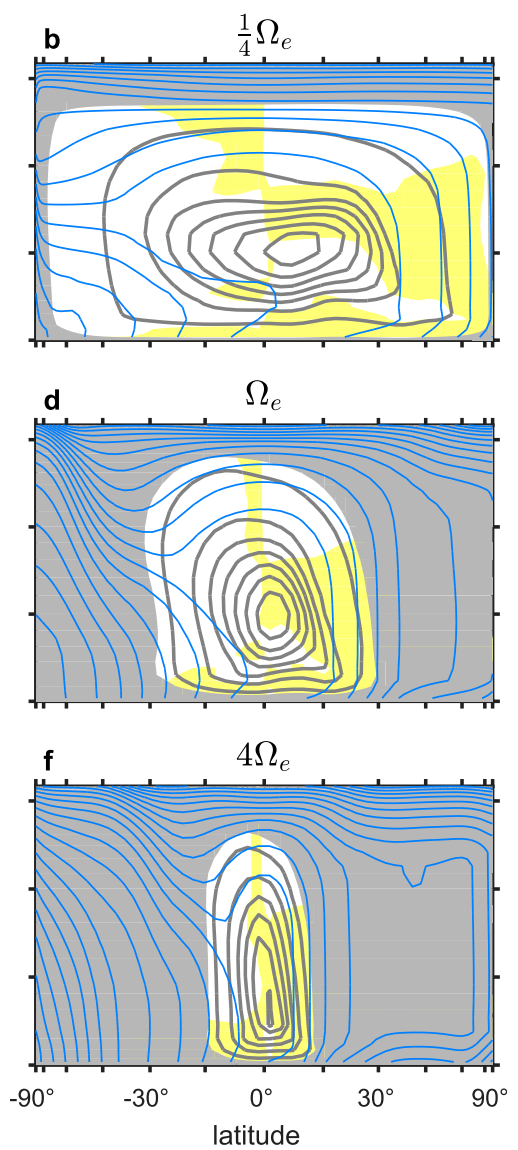

FIG. 7. Streamlines (gray lines) as a function of sine latitude in simulations with planetary rotation rates of (a),(b) $\Omega_{e} / 4\left(\mathrm{CI}=2 \times 10^{11} \mathrm{~kg} \mathrm{~s}^{-1}\right)$, (c),(d) $\Omega_{e}\left(\mathrm{CI}=1 \times 10^{11} \mathrm{~kg} \mathrm{~s}^{-1}\right)$, and (e),(f) $4 \Omega_{e}\left(\mathrm{CI}=0.5 \times 10^{11} \mathrm{~kg} \mathrm{~s}^{-1}\right)$. Regions outside the outermost streamline of the SHC, where the SHC edge is defined by (2), are shaded gray. (left) Angular momentum contours (red lines) and regions where $|1-\mathrm{Ro}|<0.5$ (yellow shading). Angular momentum is contoured for values of the planetary angular momentum at latitudes of $\ldots,-15^{\circ},-9^{\circ},-3^{\circ}, 3^{\circ}, 9^{\circ}, 15^{\circ}, \ldots$. (right) Saturation entropy contours (blue lines; $\mathrm{CI}=50 \mathrm{~J} \mathrm{~kg}^{-1} \mathrm{~K}^{-1}$ ) and regions where $\mathrm{PV}^{*}<0$ (yellow shading).

thermodynamic and dynamic state of the atmosphere. As pointed out in the previous section, the RCE state is supercritical at the equator, implying the existence of a reversal in the latitudinal gradient of angular momentum. Since such a state is inertially unstable, we expect that the resultant circulation would remove such reversals in the angular momentum gradient. However, even when the atmosphere is stable to both gravitational and inertial instability, it may nevertheless be unstable to slantwise convection along angular momentum surfaces (e.g., Bennetts and Hoskins 1979; O'Neill and Kaspi 2016). We therefore make the assumption that the effect of the large-scale circulation is to adjust the atmosphere to a state of neutrality to slantwise moist convection, in which isosurfaces of saturation entropy and angular momentum coincide (Emanuel 1983a,b). We further assume that the effect of large-scale eddies on the solsticial Hadley cell is weak, and as a result, the Hadley cell remains close to the angular momentum-conserving limit in which streamlines are parallel to angular momentum surfaces within the free troposphere (Schneider and Bordoni 2008; Bordoni and Schneider 2010).

Examination of the simulated distributions of angular momentum, saturation entropy, and the streamfunction show that the above approximations are reasonable for our simulations. For all rotation rates shown, streamlines remain almost parallel to angular momentum contours within the Hadley cell at levels above $\sigma \approx 0.85$ (Figs. 7a,c,e). We quantify the proximity of the simulations to the angular momentum-conserving limit by defining a generalized Rossby number (Ro) by

$$
1-\operatorname{Ro}=\frac{\mathbf{u} \cdot \nabla M}{\mathbf{u} \cdot \nabla M_{p}}
$$


where $\mathbf{u}=(u, v, \omega)$ is the zonal- and time-mean vector velocity, and $M=R_{e} \cos \phi\left(\Omega R_{e} \cos \phi+u\right)$ is the angular momentum per unit mass with planetary component $M_{p}=\Omega R_{e}^{2} \cos ^{2} \phi$. The definition of Ro given here differs from that of previous authors (e.g., Walker and Schneider 2006) in that it includes vertical advection of angular momentum by the mean flow. Under conditions where angular momentum contours are exactly parallel to streamlines, $\mathrm{Ro}=1$. In our simulations, a substantial fraction of the SHC rising branch is in a region for which $|1-\mathrm{Ro}|<0.5$ (Figs. 7a,c,e), indicating that the circulation is relatively close to the angular momentumconserving limit.

The right panels of Fig. 7 show that the isolines of saturation entropy are also roughly parallel to streamlines and angular momentum contours, particularly in the tropical upper troposphere, indicating a state close to neutral to slantwise moist convection. In fact, in broad regions of the Hadley cell's rising branch, the simulations exhibit slight slantwise instability. Specifically, these regions are characterized by instability to saturated motion along angular momentum surfaces, as indicated by negative values of the saturation potential vorticity (Figs. $7 \mathrm{~b}, \mathrm{~d}, \mathrm{f}$ ), defined $\mathrm{PV}^{*}=\zeta_{a} \cdot \nabla s^{*}$, where $\zeta_{a}$ and $s^{*}$ are the zonal- and time-mean absolute vorticity and saturation entropy, respectively (Korty and Schneider 2007). We speculate that this instability can be maintained because the convection scheme used in our GCM cannot eliminate slantwise instability, allowing it to build up to some degree before it is released by the resolved-scale dynamics.

Having verified that the assumptions of slantwise neutrality and angular momentum conservation are reasonable in our simulations, we now use these assumptions to relate the Hadley cell extent to the distribution of boundary layer entropy $s_{b}$ in the dynamically equilibrated simulations.

We begin with the nonlinear balance equation for the zonal wind, which we write in pressure coordinates as

$$
2 \Omega \sin \phi u+\frac{u^{2} \tan \phi}{R_{e}}=-\frac{1}{R_{e}} \frac{\partial \Phi}{\partial \phi},
$$

where $\Phi$ is the time- and zonal-mean geopotential. Taking the derivative with respect to pressure, we obtain the thermal wind relation, which may be written as (see Emanuel 1995)

$$
\frac{\tan \phi}{R_{e}^{2} \cos ^{2} \phi} \frac{\partial M^{2}}{\partial p}=\frac{\partial \alpha}{\partial \phi}
$$

where $\alpha$ is the specific volume and we have used hydrostatic balance $\partial_{p} \Phi=-\alpha$. Neglecting the contribution of condensate to density, the specific volume $\alpha$ may be expressed as a thermodynamic function of pressure $p$ and saturation entropy $s^{*}$ such that $\alpha=\alpha\left(s^{*}, p\right)$. We may therefore use the chain rule to write the meridional derivative of the specific volume as

$$
\frac{\partial \alpha}{\partial \phi}=\left(\frac{\partial \alpha}{\partial s^{*}}\right) \frac{\partial s^{*}}{\partial \phi} .
$$

In the appendix, a Maxwell-type relation is derived to show that the isobaric partial derivative in (6) may be approximately reexpressed in terms of the saturated moist adiabatic lapse rate, so that

$$
\left(\frac{\partial \alpha}{\partial s^{*}}\right)_{p}=\left(\frac{\partial T}{\partial p}\right)_{s^{*}}
$$

Substituting (7) and (6) into (5), we have

$$
\frac{\tan \phi}{R_{e}^{2} \cos ^{2} \phi} \frac{\partial M^{2}}{\partial p}=\left(\frac{\partial T}{\partial p}\right)_{s^{*}} \frac{\partial s^{*}}{\partial \phi} .
$$

The above equation is identical to (7) of Emanuel (1995).

Under the assumption of slantwise neutrality, isosurfaces of angular momentum and isosurfaces of saturation entropy are coincident, and the angular momentum may be expressed as a function of saturation entropy alone $M=M\left(s^{*}\right)$. The thermal wind relation may therefore be written as

$$
\frac{\tan \phi}{R_{e}^{2} \cos ^{2} \phi} \frac{d M^{2}}{d s^{*}} \frac{\partial s^{*}}{\partial p}=\left(\frac{\partial T}{\partial p}\right)_{s^{*}} \frac{\partial s^{*}}{\partial \phi} .
$$

Dividing the above equation by $\partial s^{*} / \partial \phi$, it may be reexpressed in terms of the slope of isentropic surfaces so that

$$
\frac{\tan \phi}{R_{e}^{2} \cos ^{2} \phi}\left(\frac{\partial \phi}{\partial p}\right)_{s^{*}}=-\frac{d s^{*}}{d M^{2}}\left(\frac{\partial T}{\partial p}\right)_{s^{*}}
$$

Here, we have used the fact that the slope of isentropic surfaces is related to the meridional and vertical gradients of saturation entropy via

$$
\left(\frac{\partial \phi}{\partial p}\right)_{s^{*}}=-\frac{d s^{* / \partial p}}{d s^{* / \partial \phi}}
$$

Integrating (9) with respect to pressure along an isosurface of saturation entropy from the boundary layer to a pressure $p$, we have

$$
\tan ^{2}(\phi)-\tan ^{2}\left(\phi_{b}\right)=-\frac{2 R_{e}^{2}\left(T_{b}-T\right)}{d M_{b}^{2} / d \phi} \frac{d s_{b}}{d \phi},
$$


where the subscript $b$ refers to variables evaluated within the boundary layer, and the derivatives on the right-hand side are evaluated at $\phi=\phi_{b}$. If we assume the zonal wind in the boundary layer is weak, we may approximate the boundary layer angular momentum by its planetary value, $M_{b}=\Omega R_{e}^{2} \cos ^{2}\left(\phi_{b}\right)$, and we have

$\tan ^{2}(\phi)=\tan ^{2}\left(\phi_{b}\right)-\left.\frac{T_{b}-T}{2 \Omega^{2} R_{e}^{2} \cos ^{3}\left(\phi_{b}\right) \sin \left(\phi_{b}\right)} \frac{\partial s_{b}}{\partial \phi}\right|_{\phi_{b}}$

Equation (10) gives the latitude of a given $M$ surface as a function of temperature, which we treat here as the vertical coordinate. The author was made aware of the preceding derivation through an unpublished manuscript (K. A. Emanuel 2011, personal communication), but a similar equation is derived for angular momentum surfaces within an axisymmetric tropical cyclone in Emanuel (1986), and the present model should be seen as the application of the same reasoning to an axisymmetric Hadley cell.

For the current solsticial case, the boundary layer entropy $s_{b}$ generally increases toward the north. By (10), this implies that angular momentum surfaces that begin in the boundary layer in the Northern Hemisphere are inclined with height toward the equator. Angular momentum surfaces calculated based on (10) and the distribution of $s_{b}$ in the simulations are shown in Fig. 8. For high planetary rotation rates, the angular momentum surfaces are approximately vertical in polar regions, but they begin to tilt toward the equator at low latitudes. For values of $\phi_{b}$ sufficiently close to the equator, the angular momentum surfaces reach $\phi=0^{\circ}$, at which point they must be horizontal to ensure that the distribution of entropy satisfies (8). More generally, angular momentum surfaces must have zero slope as they cross the equator to ensure inertial stability of the solution in the neighborhood of the equator. In the axisymmetric limit, this implies that streamlines of the SHC must also be approximately horizontal at the equator, as seen in our simulations (Fig. 3) and in previous studies with a dry, axisymmetric GCM (Caballero et al. 2008).

On the winter side of the equator, the negative slope of angular momentum surfaces implies descending motion for angular momentum-conserving flow, and the assumption of slantwise neutrality is no longer appropriate. We therefore focus only on the summer hemisphere in the present manuscript, although we note that recent work has suggested that the SHC must extend at least as far into the winter hemisphere as into the summer hemisphere (Hill et al. 2019), and our summerhemisphere solution may therefore be useful as a lower bound on the winter-hemisphere SHC extent.
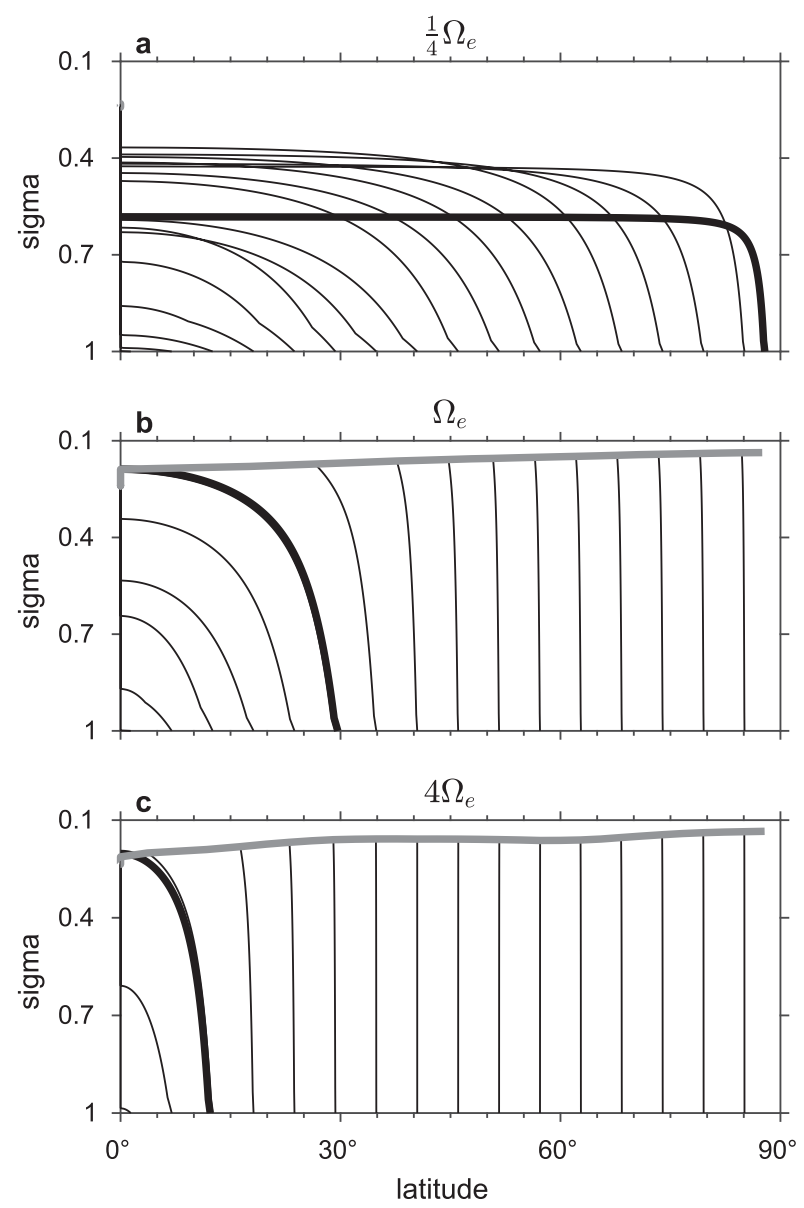

FIG. 8. Distribution of angular momentum contours for the slantwise neutral solution for planetary rotation rates of (a) $\Omega_{e} / 4$, (b) $\Omega_{e}$, and (c) $4 \Omega_{e}$. Angular momentum contours are calculated via (10) and converted to sigma coordinates by assuming neutrality to a parcel lifted reversibly from the surface along an angular momentum contour. Thick line shows the angular momentum contour corresponding to the boundary of the SHC, and gray line shows the tropopause, defined here as the location at which $\Delta T=85 \mathrm{~K}$ along each angular momentum contour. In (a) all angular momentum contours reach the equator before they reach the tropopause.

Note also that nothing in (10) prevents angular momentum surfaces that emanate from the boundary layer at different latitudes from intersecting, and this occurs in the case $\Omega=\Omega_{e} / 4$ as $\phi_{b}$ approaches the pole. As $\phi_{b}$ approaches the pole, however, both terms on the righthand side of (10) approach infinity, implying that the shape of angular momentum surfaces becomes highly sensitive to small changes in the latitudinal gradient in $s_{b}$. Thus, while the intersection of angular momentum surfaces strictly renders the solution produced by this procedure unphysical, this may simply reflect difficulty in accurately estimating the boundary layer entropy gradient or slight deviations from a state of slantwise neutrality in polar regions. 
Under the assumption that streamlines must be parallel to angular momentum surfaces, (10) places a strong constraint on the SHC. In particular, if we take the tropopause as the upper bound to the SHC, a crossequatorial cell can only exist where angular momentum surfaces that begin in the boundary layer of the Northern Hemisphere reach the equator in the free troposphere. This region is bounded by the angular momentum surface that reaches the equator at the tropopause and enters the boundary layer at the latitude $\phi_{s}$, given by

$$
\sin ^{3}\left(\phi_{s}\right) \cos \left(\phi_{s}\right)=\left.\frac{\Delta T}{2 \Omega^{2} R_{e}^{2}} \frac{\partial s_{b}}{\partial \phi}\right|_{\phi_{s}},
$$

where $\Delta T=T_{b}-T_{t}$ is the difference between the temperature at the equatorial tropopause $T_{t}$ and the temperature within boundary layer at $\phi_{s}$.

Given the value of $\Delta T$, the Hadley cell extent may be estimated by calculating the latitude $\phi_{s}$ using (11). In the simulations, we estimate $\Delta T$ as the difference between the temperature at the lowest model level at the latitude of the SHC edge $\phi_{h}$ and the minimum temperature on the outermost streamline of the SHC. Under this definition, $\Delta T \approx 85 \mathrm{~K}$ for all rotation rates $^{6}$ (Fig. 9). The reason for this insensitivity of $\Delta T$ across such a wide range of circulations is unclear at present, and it is likely to be affected by the relative simplicity of the radiation scheme we have used [cf. Hartmann and Larson (2002), who argued that longwave emission by water vapor constrains the height of convective clouds to remain at fixed temperature even as the surface temperature varies]. For the present purposes, however, we take $\Delta T=85 \mathrm{~K}$ as fixed in the calculations below.

In principle, there may be more than one latitude where (11) is satisfied, but we find that $\phi_{s}$, if it exists, is unique in our set of simulations. On the other hand, for the slowly rotating cases, $\Omega \leq \Omega_{e} / 4$, all angular momentum contours reach the equator before they reach the temperature $T_{t}$; we interpret this as a Hadley cell that extends to the summer pole. Under this interpretation, the slantwise neutral solution provides an excellent estimate for the simulated SHC edge latitude $\phi_{h}$ at all rotation rates (Figs. 4 and 10), although the precise value of $\phi_{h}$ depends somewhat on the threshold used in (2). Note also that, according to the slantwise neutral model, streamlines above the boundary layer must be horizontal as they cross the equator, implying that the

\footnotetext{
${ }^{6}$ If $T_{b}$ is calculated based on an average below $\sigma=0.8$ rather than at the lowest model level, this value is reduced somewhat $(\Delta T \approx 80 \mathrm{~K})$, but this has a negligible effect on the prediction of the SHC edge $\phi_{s}$.
}

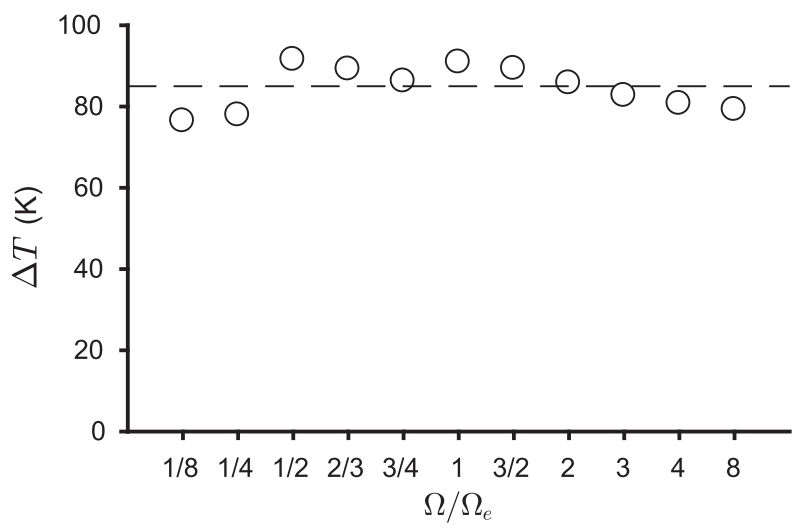

FIG. 9. Temperature difference $\Delta T=T_{b}-T_{t}$ for all simulations. Here $T_{b}$ is taken as the zonal- and time-mean temperature at the first model level at the latitude $\phi_{h}$, and $T_{t}$ is taken as the lowest temperature on the outermost streamline of the SHC. Dashed line shows the value of $85 \mathrm{~K}$.

streamfunction maximum remains at the equator even as the SHC edge shifts toward the pole. This is consistent with the GCM simulations, in which the streamfunction maximum remains between $0^{\circ}$ and $10^{\circ}$ in all cases.

The close correspondence between $\phi_{s}$ as predicted by the slantwise neutral solution and $\phi_{h}$ the simulated SHC extent suggests that boundary layer entropy distribution places a useful constraint on the position of the summerhemisphere SHC edge. Previous studies based on convective quasi equilibrium have argued that rain belts are constrained to remain close to tropical maxima in boundary layer entropy. In our simulations, the boundary layer entropy increases monotonically toward the pole, and so there is no local maximum in $s_{b}$. Nevertheless, by considering the case in which a maximum in $s_{b}$ exists at low latitudes of the summer hemisphere, we may show that (11) represents a generalization of these previous quasi-equilibrium constraints. Consider (11) under the small angle approximation,

$$
\phi_{s}^{3}=\left.\frac{\Delta T}{2 \Delta T_{E}}\left(\frac{\Omega_{e}}{\Omega}\right)^{2} \frac{\partial \hat{s}_{b}}{\partial \phi}\right|_{\phi_{s}},
$$

where we have nondimensionalized the entropy as in section 4 . We expect the gradient $\partial_{\phi} \hat{s}_{b}$ to decrease from a positive value at the equator to zero at the latitude of the $\hat{s}_{b}$ maximum. By the above equation, $\phi_{s}$ must be equatorward of the maximum in $\hat{s}_{b}$. Furthermore, at low latitudes, $\phi_{s}^{3} \ll 1$, and $\phi_{s}$ must occur in the region where $\partial_{\phi} \hat{s}_{b}$ is small. Provided the latitudinal gradient $\partial_{\phi} \hat{s}_{b}$ is of order unity at the equator, this will only occur in the vicinity of the latitudinal maximum in $s_{b}$. The value of $\phi_{s}$ is therefore well approximated by the location of the 


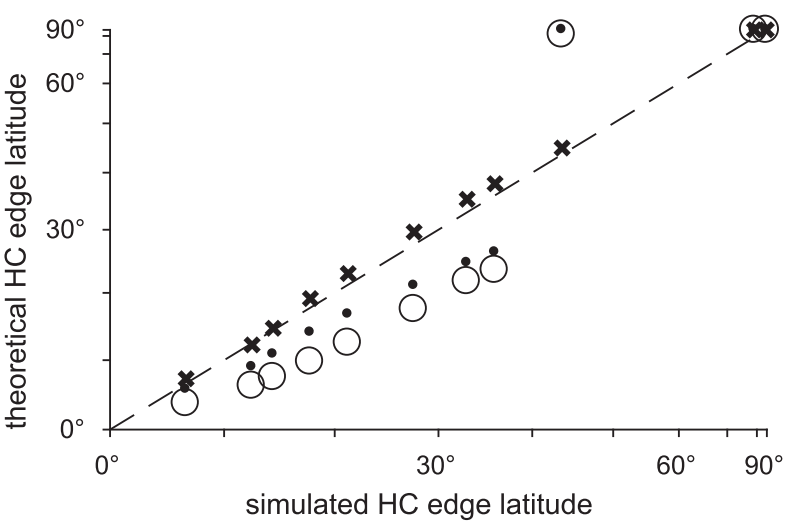

FIG. 10. Theoretical predictions of the Hadley cell extent based on supercriticality of the RCE state (circles) and slantwise neutral solution based on RCE state (dots) and equilibrated simulations (crosses) plotted against simulated Hadley cell extent for rotation rates between $\Omega_{e} / 8$ and $8 \Omega_{e}$. One-to-one line is also shown (dashed)

boundary layer entropy maximum when this maximum is relatively close to the equator.

The slantwise neutral theory presented in this section provides a diagnostic for the Hadley cell extent under perpetual-solstice conditions. To formulate a fully closed theory, a prediction for the boundary layer entropy distribution is required. The distribution of $s_{b}$ is determined by the energy balance of the boundary layer, and, in particular, it depends on the energy transport by the atmospheric circulation. A full theory of the energy transport by the Hadley cell is beyond the scope of the current work. However, some insight may be gained by considering the limiting case in which the Hadley cell does not transport energy. Under these conditions, the boundary layer entropy distribution would be approximately equal to that of the RCE solution $s_{E}$, and, assuming slantwise neutrality, the Hadley cell extent would be obtained by applying (11) to the RCE entropy distribution. This prediction of the SHC extent gives a slightly improved estimate of the SHC extent in comparison to the supercriticality-based prediction, but it is still too far equatorward at most rotation rates (Fig. 10). ${ }^{7}$

In the simulations, the energy transport by the SHC is from the Northern to the Southern Hemisphere, and we would expect it to have the effect of reducing $s_{b}$ in the region of the ascending branch. Furthermore, to the extent that the SHC itself dominates the atmospheric energy transport, we would expect $s_{b} \approx s_{E}$ northward of

\footnotetext{
${ }^{7}$ When applied to the RCE entropy distribution, the slantwise neutral solution predicts more than one latitude that satisfies (11) for some rotation rates. In these cases, we take the poleward most latitude as our prediction of the $\mathrm{HC}$ edge latitude.
}

the SHC (Figs. 3d-f). The effect of the SHC energy transport on the distribution of boundary layer entropy would therefore be to increase the meridional gradient of $s_{b}$ near the Hadley cell edge (Schneider and Bordoni 2008). By (11), this would push the SHC edge toward the pole.

Based on the above reasoning, we expect the summerhemisphere SHC extent to be greater than that predicted based on the RCE entropy distribution (except when the RCE distribution predicts a global SHC), and this is indeed what is seen in the simulations (Fig. 10). Furthermore, this reasoning suggests that the extent to which the SHC edge exceeds the RCE prediction depends on the strength of the energy transport associated with the cell itself. According to this view, the SHC extent is determined in part by the strength of the SHC, and these two aspects must be solved for together in any complete theory of the Hadley circulation.

\section{Conclusions}

Using a set of idealized GCM simulations run under perpetual-solstice conditions, we have investigated the limits placed by planetary rotation on the penetration of the solsticial Hadley cell into the summer hemisphere. Consistent with previous work (Caballero et al. 2008; Faulk et al. 2017), the SHC expands as the planetary rotation rate is reduced, and at low enough values of the planetary rotation rate, the circulation is global. At Earthlike planetary rotation, however, the Hadley cell extends only roughly $30^{\circ}$ into the summer hemisphere, suggesting that rotation may play an important role in modulating the poleward extent of Earth's monsoon circulations.

We developed two theoretical frameworks through which to interpret our numerical results. The first theory provides a prediction of the SHC extent by assuming that the Hadley cell exists only in regions of the atmosphere for which an unattainable distribution of angular momentum would be required to maintain RCE (Plumb and Hou 1992; Emanuel 1995). This theory qualitatively reproduces the behavior of the simulations, but it generally underestimates the simulated SHC extent. We therefore argue that the RCE state and its angular momentum distribution may provide a useful lower bound on estimates of the extent of the Hadley cell (see also Hill et al. 2019).

A more quantitatively accurate estimate of the summer hemisphere SHC extent was obtained by assuming that, in the region of the SHC rising branch, the atmosphere adjusts toward a state neutral to slantwise convection. Under the additional assumption that angular 
momentum is conserved along streamlines, this allows for a determination of the Hadley cell extent and the shape of angular momentum contours in the summer hemisphere. This diagnostic estimate of the SHC extent quantitatively reproduces the behavior of the simulations, and it connects the summer-hemisphere SHC extent to the distribution of boundary layer entropy. Previous work based on the assumption of convective quasi equilibrium has also highlighted the importance of the boundary layer entropy distribution in determining the position of tropical rain belts (Neelin and Held 1987; Emanuel 1995; Nie et al. 2010; Hurley and Boos 2013); the diagnostic theory presented here may be considered a generalization of this convective quasi-equilibrium view to allow for slantwise convection adjustment.

While the slantwise neutral theory accurately reproduces the behavior of the summer-hemisphere SHC extent, we have not addressed the dependence of precipitation belts on rotation rate in detail. In our simulations, the latitude of maximum precipitation $\phi_{P}$ behaves nonmonotonically with rotation rate, and in many simulations there are at least two precipitation maxima of similar magnitude in the zonal- and timemean precipitation field. Faulk et al. (2017) argued that the localization of precipitation into intense convergence zones such as the ITCZ should be understood through the boundary layer momentum budget, which we do not consider here. Nevertheless, the summerhemisphere extent of the SHC provides a good measure of the latitudinal extent of the region of strong moisture convergence in our simulations, and thus the latitudinal edge of the SHC is an important parameter for understanding the tropical and subtropical hydrological cycle.

The extent of the SHC in our perpetual-solstice simulations should be seen as an upper limit on the poleward seasonal migration of the Hadley cell in which the atmospheric heat capacity plays no role. In Earth's atmosphere, transient effects are important in limiting the poleward migration of the circulation in summer, particularly in oceanic regions, making direct application of our results to observations difficult. Nonetheless, previous studies have noted that, in the summer season, Earth's cross-equatorial Hadley circulation more closely approaches the angular momentum-conserving limit (Bordoni and Schneider 2008), and that, in the region of the Asian monsoon, the upper-tropospheric potential vorticity becomes small (e.g., Plumb 2007). These features point to the potential relevance of the slantwise neutral solution to the Asian summer monsoon. Determining the extent to which a similar constraint may relate the poleward extent of the Asian summer monsoon to the low-level entropy distribution is an interesting avenue for future work.

Acknowledgments. I thank K. A. Emanuel, who brought the slantwise neutral solution to my attention in a graduate class on tropical meteorology, and S. A. Hill and two anonymous reviewers for their constructive comments. I acknowledge support from the ARC Centre of Excellence for Climate Extremes, support from an ARC Discovery Early Career Research Award (DE190100866), and computational support from the National Computational Infrastructure, all funded by the Australian Government.

\section{APPENDIX}

\section{Derivation of Maxwell's Relation}

Here, we derive the Maxwell-type relation (7). This differs from the usual Maxwell relationship for entropy and specific volume (see Emanuel 1994, chapter 4) because it is written in terms of saturation entropy $s^{*}$, which is only a function of temperature and pressure, rather than in terms of entropy, which is a function of temperature, pressure, and total water content.

Consider the thermodynamic functions enthalpy $k$ and entropy $s$, defined at saturation,

$$
\begin{aligned}
& k^{*}=\left[\left(1-q_{v}^{*}\right) c_{p d}+q_{v}^{*} c_{p v}\right]\left(T-T_{0}\right)+L_{v 0} q_{v}^{*}, \\
& s^{*}=\left[\left(1-q_{v}^{*}\right) c_{p d}+q_{v}^{*} c_{p v}\right] \log \left(\frac{T}{T_{0}}\right)+\frac{L_{v 0} q_{v}^{*}}{T_{0}} \\
& -\left(1-q_{v}^{*}\right) R_{d} \log \left(\frac{p-e^{*}}{e_{0}}\right)-q_{v}^{*} R_{v} \log \left(\frac{e^{*}}{e_{0}}\right) .
\end{aligned}
$$

Here, $q_{v}$ is the specific humidity, $e$ is the vapor pressure, $c_{p d}$ and $c_{p v}$ are the isobaric specific heat capacities of dry air and water vapor, respectively, and a superscript asterisk refers to a variable at saturation. Additionally, we have defined $L_{v 0}$ and $e_{0}$ as the latent enthalpy of vaporization and the saturation vapor pressure, both defined at the reference temperature $T_{0}$. Approximating all specific heat capacities as constant and taking the derivative of the saturation enthalpy, we have

$$
\begin{aligned}
d k^{*}= & {\left[\left(1-q_{v}^{*}\right) c_{p d}+q_{v}^{*} c_{p v}\right] d T } \\
& +\left[L_{v}+\left(c_{p l}-c_{p d}\right)\left(T-T_{0}\right)\right] d q_{v}^{*}
\end{aligned}
$$

where $L_{v}=L_{v 0}+\left(c_{p v}-c_{p l}\right)\left(T-T_{0}\right)$ is the latent enthalpy of vaporization, and $c_{p l}$ is the specific heat capacity 
of liquid water. The derivative of saturation entropy may be written as

$$
\begin{aligned}
d s^{*}= & {\left[\left(1-q_{v}^{*}\right) c_{p d}+q_{v}^{*} c_{p v}\right] \frac{d T}{T}-\alpha \frac{d p}{T} } \\
& +\left[\frac{L_{v 0}}{T_{0}}+\left(c_{p v}-c_{p d}\right) \log \left(\frac{T}{T_{0}}\right)-R_{v} \log \left(\frac{e^{*}}{e_{0}}\right)\right] d q_{v}^{*} .
\end{aligned}
$$

Using the Clausius-Clapeyron relation, the saturation vapor pressure may be written as

$$
\begin{aligned}
\log \left(\frac{e^{*}}{e_{0}}\right)= & \left(\frac{c_{p v}-c_{p l}}{R_{v}}\right) \log \left(\frac{T}{T_{0}}\right) \\
& +\left[\frac{L_{v 0}-T_{0}\left(c_{p v}-c_{p l}\right)}{R_{v}}\right]\left(\frac{1}{T_{0}}-\frac{1}{T}\right) .
\end{aligned}
$$

Substituting this into (A2), we have

$$
\begin{aligned}
T d s^{*}= & {\left[\left(1-q_{v}^{*}\right) c_{p d}+q_{v}^{*} c_{p v}\right] d T-\alpha d p } \\
& +L_{v} d q_{v}^{*}-\left(c_{p l}-c_{p d}\right) \log \left(\frac{T}{T_{0}}\right) d q_{v}^{*} .
\end{aligned}
$$

Combining (A1) and (A3), we may write

$$
\begin{aligned}
d k^{*}= & T d s^{*}+\alpha d p \\
& +\left(c_{p l}-c_{p d}\right)\left[\left(T-T_{0}\right)-T \log \left(\frac{T}{T_{0}}\right)\right] d q_{v}^{*} .
\end{aligned}
$$

The term in square brackets in the above equation vanishes to first order in $\left(T-T_{0}\right) / T_{0}$, suggesting it may be neglected. Indeed, approximate evaluation of the terms in (A4) using finite differences reveals that the term proportional to $d q_{v}^{*}$ is at least two orders of magnitude smaller than the sum of the other two terms on the right-hand side. We therefore have the following approximate relationships for the partial derivatives of the enthalpy,

$$
\begin{gathered}
\left(\frac{\partial k^{*}}{\partial s^{*}}\right)_{p} \approx T, \\
\left(\frac{\partial k^{*}}{\partial p}\right)_{s^{*}} \approx \alpha .
\end{gathered}
$$

Cross differentiating the above equations and equating the result, we obtain the desired relation. Note that the approximation made in obtaining these relationships from (A4) is equivalent to neglecting the difference between the reversible and pseudoadiabatic lapse rates.

\section{REFERENCES}

Back, L. E., and C. S. Bretherton, 2009a: On the relationship between SST gradients, boundary layer winds, and convergence over the tropical oceans. J. Climate, 22, 4182-4196, https:// doi.org/10.1175/2009JCLI2392.1.

— vertical motion patterns over the tropical oceans. J. Climate, 22, 6477-6497, https://doi.org/10.1175/2009JCLI2393.1.

Bennetts, D. A., and B. J. Hoskins, 1979: Conditional symmetric instability-A possible explanation for frontal rainbands. Quart. J. Roy. Meteor. Soc., 105, 945-962, https://doi.org/10.1002/ qj. 49710544615.

Bischoff, T., and T. Schneider, 2014: Energetic constraints on the position of the intertropical convergence zone. J. Climate, 27, 4937-4951, https://doi.org/10.1175/JCLI-D-13-00650.1.

Bordoni, S., and T. Schneider, 2008: Monsoons as eddy-mediated regime transitions of the tropical overturning circulation. Nat. Geosci., 1, 515-519, https://doi.org/10.1038/ngeo248.

- , and - 2010: Regime transitions of steady and timedependent Hadley circulations: Comparison of axisymmetric and eddy-permitting simulations. J. Atmos. Sci., 67, 16431654, https://doi.org/10.1175/2009JAS3294.1.

Caballero, R., 2007: Role of eddies in the interannual variability of Hadley cell strength. Geophys. Res. Lett., 34, L22705, https:// doi.org/10.1029/2007GL030971.

_ 2008: Hadley cell bias in climate models linked to extratropical eddy stress. Geophys. Res. Lett, 35, L18709, https:// doi.org/10.1029/2008GL035084.

— R. T. Pierrehumbert, and J. L. Mitchell, 2008: Axisymmetric, nearly inviscid circulations in non-condensing radiative-convective atmospheres. Quart. J. Roy. Meteor. Soc., 134, 1269-1285, https://doi.org/10.1002/qj.271.

Dima, I. M., and J. M. Wallace, 2003: On the seasonality of the Hadley cell. J. Atmos. Sci., 60, 1522-1527, https://doi.org/ 10.1175/1520-0469(2003)060<1522:OTSOTH>2.0.CO;2.

Donohoe, A., J. Marshall, D. Ferreira, and D. McGee, 2013: The relationship between ITCZ location and cross-equatorial atmospheric heat transport: From the seasonal cycle to the Last Glacial Maximum. J. Climate, 26, 3597-3618, https://doi.org/ 10.1175/JCLI-D-12-00467.1.

—, D. M. W. Frierson, and D. S. Battisti, 2014: The effect of ocean mixed layer depth on climate in slab ocean aquaplanet experiments. Climate Dyn., 43, 1041-1055, https://doi.org/ 10.1007/s00382-013-1843-4.

Emanuel, K. A., 1983a: The Lagrangian parcel dynamics of moist symmetric instability. J. Atmos. Sci., 40, 2368-2376, https://doi.org/ 10.1175/1520-0469(1983)040<2368:TLPDOM >2.0.CO;2.

_- 1983b: On assessing local conditional symmetric instability from atmospheric soundings. Mon. Wea. Rev., 111, 2016-2033, https://doi.org/10.1175/1520-0493(1983) $111<2016$ :OALCSI $>2.0$.CO;2.

1986: An air-sea interaction theory for tropical cyclones. Part I: Steady-state maintenance. J. Atmos. Sci., 43, 585604, https://doi.org/10.1175/1520-0469(1986)043<0585: AASITF $>2.0 . \mathrm{CO} ; 2$.

— 1988: Observational evidence of slantwise convective adjustment. Mon. Wea. Rev., 116, 1805-1816, https://doi.org/ 10.1175/1520-0493(1988)116<1805:OEOSCA > 2.0.CO;2. , 1994: Atmospheric Convection. Oxford University Press, 580 pp. , 1995: On thermally direct circulations in moist atmospheres. J. Atmos. Sci., 52, 1529-1534, https://doi.org/10.1175/15200469(1995)052<1529:OTDCIM>2.0.CO;2. 
_ J. D. Neelin, and C. S. Bretherton, 1994: On large-scale circulations in convecting atmospheres. Quart. J. Roy. Meteor. Soc., 120, 1111-1143, https://doi.org/10.1002/qj.49712051902.

Faulk, S., J. Mitchell, and S. Bordoni, 2017: Effects of rotation rate and seasonal forcing on the ITCZ extent in planetary atmospheres. J. Atmos. Sci., 74, 665-678, https://doi.org/10.1175/ JAS-D-16-0014.1.

Frierson, D. M. W., 2007: The dynamics of idealized convection schemes and their effect on the zonally averaged tropical circulation. J. Atmos. Sci., 64, 1959-1976, https://doi.org/10.1175/ JAS3935.1.

— I. M. Held, and P. Zurita-Gotor, 2006: A gray-radiation aquaplanet moist GCM. Part I: Static stability and eddy scale. J. Atmos. Sci., 63, 2548-2566, https://doi.org/10.1175/JAS3753.1.

$\longrightarrow,-$, and $\longrightarrow, 2007$ : A gray-radiation aquaplanet moist GCM. Part II: Energy transports in altered climates. J. Atmos. Sci., 64, 1680-1693, https://doi.org/10.1175/JAS3913.1.

Hartmann, D. L., 1994: Global Physical Climatology. Academic Press, $411 \mathrm{pp}$.

— , and K. Larson, 2002: An important constraint on tropical cloud-climate feedback. Geophys. Res. Lett., 29, 1951, https:// doi.org/10.1029/2002GL015835.

Held, I. M., and A. Y. Hou, 1980: Nonlinear axially symmetric circulations in a nearly inviscid atmosphere. J. Atmos. Sci., 37, 515-533, https://doi.org/10.1175/1520-0469(1980)037<0515: NASCIA $>2.0 . \mathrm{CO} ; 2$.

Hide, R., 1969: Dynamics of the atmospheres of the major planets with an appendix on the viscous boundary layer at the rigid bounding surface of an electrically-conducting rotating fluid in the presence of a magnetic field. J. Atmos. Sci., 26, 841-853, https://doi.org/ 10.1175/1520-0469(1969)026<0841:DOTAOT >2.0.CO;2.

Hill, S. A., S. Bordoni, and J. L. Mitchell, 2019: Axisymmetric constraints on cross-equatorial Hadley cell extent. J. Atmos. Sci., 76, 1547-1564, https://doi.org/10.1175/JAS-D-18-0306.1.

Hurley, J. V., and W. R. Boos, 2013: Interannual variability of monsoon precipitation and local subcloud equivalent potential temperature. J. Climate, 26, 9507-9527, https://doi.org/ 10.1175/JCLI-D-12-00229.1.

Kang, S. M., I. M. Held, D. M. W. Frierson, and M. Zhao, 2008: The response of the ITCZ to extratropical thermal forcing: Idealized slab-ocean experiments with a GCM. J. Climate, 21, 35213532, https://doi.org/10.1175/2007JCLI2146.1.

, D. M. W. Frierson, and I. M. Held, 2009: The tropical response to extratropical thermal forcing in an idealized GCM: The importance of radiative feedbacks and convective parameterization. J. Atmos. Sci., 66, 2812-2827, https://doi.org/ 10.1175/2009JAS2924.1.

Korty, R., and T. Schneider, 2007: A climatology of the tropospheric thermal stratification using saturation potential vorticity. J. Climate, 20, 5977-5991, https://doi.org/10.1175/ 2007JCLI1788.1.

Lindzen, R. S., and S. Nigam, 1987: On the role of sea surface temperature gradients in forcing low-level winds and convergence in the tropics. J. Atmos. Sci., 44, 2418-2436, https://doi.org/ 10.1175/1520-0469(1987)044<2418:OTROSS > 2.0.CO;2.

— aged heating centered off the equator. J. Atmos. Sci., 45, 2416-2427, https://doi.org/10.1175/1520-0469(1988)045<2416: $\mathrm{HCFZAH}>2.0 . \mathrm{CO} ; 2$.

Neelin, J. D., and I. M. Held, 1987: Modeling tropical convergence based on the moist static energy budget. Mon. Wea. Rev., 115, 3-12, https://doi.org/10.1175/1520-0493(1987)115<0003: MTCBOT>2.0.CO;2.
Nie, J., W. R. Boos, and Z. Kuang, 2010: Observational evaluation of a convective quasi-equilibrium view of monsoons. J. Climate, $\mathbf{2 3}, 4416-4428$, https://doi.org/10.1175/2010JCLI3505.1.

O'Gorman, P. A., and T. Schneider, 2008: The hydrological cycle over a wide range of climates simulated with an idealized GCM. J. Climate, 21, 3815-3832, https://doi.org/10.1175/ 2007JCLI2065.1.

O'Neill, M. E., and Y. Kaspi, 2016: Slantwise convection on fluid planets. Geophys. Res. Lett., 43, 10 611-10 620, https://doi.org/ 10.1002/2016GL071188.

Plumb, R. A., 2007: Dynamical constraints on monsoon circulations. The Global Circulation of the Atmosphere, T. Schneider and A. H. Sobel, Eds., Princeton University Press, 252-266.

_ , and A. Y. Hou, 1992: The response of a zonally symmetric atmosphere to subtropical thermal forcing: Threshold behavior. J. Atmos. Sci., 49, 1790-1799, https://doi.org/10.1175/15200469(1992)049<1790:TROAZS >2.0.CO;2.

Privé, N. C., and R. A. Plumb, 2007a: Monsoon dynamics with interactive forcing. Part I: Axisymmetric studies. J. Atmos. Sci., 64, 1417-1430, https://doi.org/10.1175/JAS3916.1.

— , and $-2007 \mathrm{~b}$ : Monsoon dynamics with interactive forcing. Part II: Impact of eddies and asymmetric geometries. J. Atmos. Sci., 64, 1431-1442, https://doi.org/10.1175/ JAS3917.1.

Schneider, E. K., 1977: Axially symmetric steady-state models of the basic state for instability and climate studies. Part II. Nonlinear calculations. J. Atmos. Sci., 34, 280-296, https://doi.org/10.1175/ 1520-0469(1977)034<0280:ASSSMO > 2.0.CO;2.

Schneider, T., and S. Bordoni, 2008: Eddy-mediated regime transitions in the seasonal cycle of a Hadley circulation and implications for monsoon dynamics. J. Atmos. Sci., 65, 915-934, https://doi.org/10.1175/2007JAS2415.1.

Shekhar, R., and W. R. Boos, 2016: Improving energy-based estimates of monsoon location in the presence of proximal deserts. J. Climate, 29, 4741-4761, https://doi.org/10.1175/JCLI-D-150747.1.

Singh, M. S., and Z. Kuang, 2016: Exploring the role of eddy momentum fluxes in determining the characteristics of the equinoctial Hadley circulation: Fixed-SST simulations. J. Atmos. Sci., 73, 2427-2444, https://doi.org/10.1175/JAS-D-15-0212.1.

- — - and Y. Tian, 2017: Eddy influences on the strength of the Hadley circulation: Dynamic and thermodynamic perspectives. J. Atmos. Sci., 74, 467-486, https://doi.org/10.1175/ JAS-D-16-0238.1.

Sobel, A. H., J. Nilsson, and L. M. Polvani, 2001: The weak temperature gradient approximation and balanced tropical moisture waves. J. Atmos. Sci., 58, 3650-3665, https://doi.org/ 10.1175/1520-0469(2001)058<3650:TWTGAA > 2.0.CO;2.

Troen, I. B., and L. Mahrt, 1986: A simple model of the atmospheric boundary layer; sensitivity to surface evaporation. Bound.-Layer Meteor., 37, 129-148, https://doi.org/10.1007/ BF00122760.

Walker, C. C., and T. Schneider, 2006: Eddy influences on Hadley circulations: Simulations with an idealized GCM. J. Atmos. Sci., 63, 3333-3350, https://doi.org/10.1175/JAS3821.1.

Wang, B., and Q. Ding, 2008: Global monsoon: Dominant mode of annual variation in the tropics. Dyn. Atmos. Oceans, 44, 165183, https://doi.org/10.1016/j.dynatmoce.2007.05.002.

Wei, H.-H., and S. Bordoni, 2018: Energetic constraints on the ITCZ position in idealized simulations with a seasonal cycle. J. Adv. Model. Earth Syst., 10, 1708-1725, https://doi.org/ 10.1029/2018MS001313. 